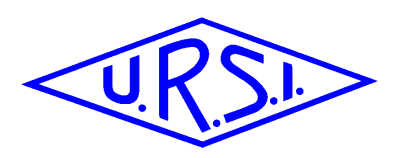

\title{
Low Power High-Speed Folding ADC based Partial Discharge Sensor for Wireless Fault Detection in Substations
}

\author{
D. W. Upton*(1), R. P. Haigh ${ }^{(1)}$, B. I. Saeed ${ }^{(1)}, U$ Khan $^{(1)}$, H. Mohamed ${ }^{(1)}$, K. Mistry ${ }^{(1)}$, P. J. Mather ${ }^{(1)}$, P. I. Lazaridis ${ }^{(1)}$, F. \\ Torres Filho ${ }^{(2)}$, C. Tachtatzis ${ }^{(3)}$, R. Atkinson ${ }^{(3)}$, M. Judd ${ }^{(4)}$, and I. A. Glover ${ }^{(1)}$ \\ (1) Department of Engineering and Technology, University of Huddersfield, Huddersfield HD1 3DH, UK \\ (2) Instituto Federal da Paraiba / Campus Patos, Department of Electrotechnics, 58700-000 Patos, PB, Brazil \\ (3) Department of Electronic and Electrical Engineering, University of Strathclyde, Glasgow G1 1XQ, UK
}

(4) High Frequency Diagnostics and Engineering Ltd., Glasgow G14 0BX, UK

\begin{abstract}
Partial discharge is a well-established metric for condition monitoring and assessment of high-voltage plant equipment, such as switchgear, transformers and transmission lines. Traditional techniques for the detection of partial discharge pulses involve physical connection of sensors to the device under observation, requiring separate sensors for each item of plant, which can be costly and time consuming. Wireless partial discharge measurement provides an attractive alternative, since sensors can monitor multiple items of plant simultaneously. However, direct sampling of the received UHF partial discharge pulse is unsuitable for a scalable wireless sensor network, due to the high cost and power consumption required for high-speed data conversion. A sensor is proposed that utilizes a pipelined, sample-and-hold based, folding analogue to digital converter structure that features low power consumption when no signal is present, making it suitable for low-power data conversion of radiometric partial discharge. A proof of concept circuit has been developed using discrete components to evaluate the performance of the system with 8-bit resolution at 25 $\mathrm{MSa} / \mathrm{s}$ and $56 \mathrm{~mW}$ of power consumption.
\end{abstract}

\section{Introduction}

Partial discharge (PD) is a fault that occurs within the insulating material of high-voltage (HV) equipment. PD is defined by the IEC60270 standard as "localized electrical discharge that only partially bridges the insulation between conductors and which can or cannot occur adjacent to a conductor. Partial discharges are in general a consequence of local electrical stress concentrations in the insulation or on the surface of the insulation. Generally, such discharges appear as pulses having a duration of much less than 1 microsecond" [1]. Traditional methods of detecting and monitoring PD, such as high-frequency current transformers (HFCT) and transient earth voltage (TEV) sensors [2-6], provide detailed information of the PD events under measurement, such as apparent charge and frequency spectra, allowing for evaluation of the PD fault type and progression assessment to be evaluated [7].
However, these techniques are only capable of monitoring local sources of PD within a single piece of plant, due to the close coupling required between the sensor and the equipment under test. Thus, separate sensors are required for each piece of plant to be monitored, which limits the scalability of such a network due to cost and complexity.

Radiometric PD monitoring avoids these limitations, as sensors are not restricted to monitoring single items of plant, making a system simpler and cheaper to install and scale. Traditional radiometric techniques, such as time-ofarrival (TOA) and time-difference-of-arrival (TDOA) [813] provide accurate wireless location estimation, but require high-speed analogue-to-digital (ADC) sampling rates in excess of $1 \mathrm{GSa} / \mathrm{s}$ in order to resolve the minute time differences between signals received at each sensor. Synchronization is also required between sensors, making implementation on a large-scale difficult.

A sensor node based on received signal-strength (RSS) only has the benefits of ease of installation, unsynchronized operation and a lower required conversion sample-rate; therefore, making a fully-scalable RSS-based wireless sensor network (WSN) an attractive solution to large-scale PD detection and monitoring. Various RSSbased PD sensors have been reported previously [14-20] which utilize envelope responding power detectors to remove the high frequency component of the received radiometric $\mathrm{PD}$. Thus, the required conversion rate is dramatically reduced. However, for acceptable accuracy a sample-rate of at least $20 \mathrm{MSa} / \mathrm{s}$ is required [21], which still places some constraints on power consumption.

Proposed is a radiometric sensor, intended for use in a large-scale PD monitoring WSN, that uses a high-speed sample-and-hold $(\mathrm{S} / \mathrm{H})$ based folding $\mathrm{ADC}$ for data conversion. The ADC is designed to only consume significant power when a PD pulse is received.

\section{Folding ADC Architecture}

The folding ADC architecture consists of a cascade of folding amplifiers (FA) which each provide a non-linear 
response that folds the input signal around half the input range. Each folding stage contains a comparator to determine which region the input signal is within, providing a Gray-coded bit-per-stage conversion process [22], as shown in Figure 1.

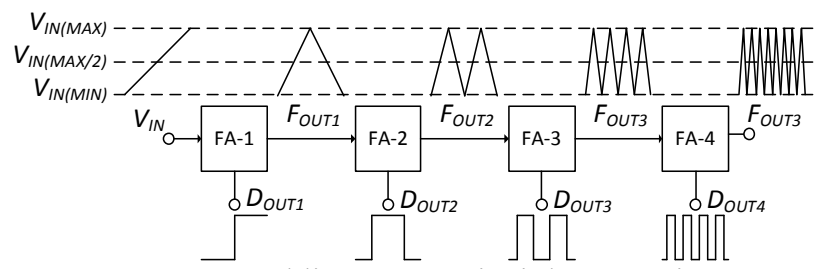

Figure 1: Folding ADC Principle Operation.

Due to this bit-per-stage response, folding ADCs typically feature low complexity compared to other high-speed architectures [23-24], such as pipelined and flash ADCs, and are therefore lower in cost and power consumption. A restriction with the standard folding $\mathrm{ADC}$ is the speed at which a signal needs to propagate through the seriesconnected FA stages, requiring high bias currents for highspeed operation, and therefore, high power consumption. This is addressed with the inclusion of $\mathrm{S} / \mathrm{H}$ sections in between FA stages to reduce the required response time to that of a single stage [25]. However, this is at an increase in circuit complexity due to the addition of $\mathrm{S} / \mathrm{H}$ amplifiers. The ADC architecture utilized in the proposed radiometric $\mathrm{PD}$ sensor incorporates the $\mathrm{S} / \mathrm{H}$ function within the FA stages, therefore reducing the required power consumption and circuit complexity. The input signal is applied to a $\mathrm{S} / \mathrm{H}$ amplifier which drives a series of $7 \mathrm{~S} / \mathrm{H} \mathrm{FAs}$. Each stage is clocked from the FPGA by a complementary clock signal, which ripples the signal through each pipelined S/H FA. The LSB is obtained using a comparator. The FAs and comparator are referenced to half the input range. The output of each stage is clocked into the FPGA, which contains alignment logic to adjust each bit to the correct sample, and Gray-code to binary conversion.

\section{Proposed PD Sensor}

The proposed high-speed folding ADC-based wireless PD sensor, shown in Figure 2, comprises of a RF front-end section, a signal processing section, a micro-controller and a wirelessHART mote for data transmission.

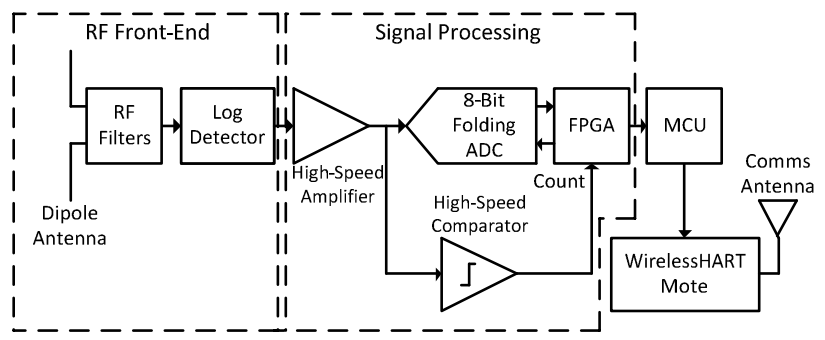

Figure 2: High-Speed $\bar{A} \bar{D} \bar{C}$-based Wireless Sensor Block Diagram.

The RF front-end is composed of a dipole antenna, bandpass and band-stop filters, a logarithmic power detector and a high-speed amplifier. The radiometric PD signal is received via the dipole antenna, which is selected since an omni-directional response is required. The band-pass filter limits the measurement range of the sensor to $30-320 \mathrm{MHz}$, whilst the band-stop filter removes potential interference from locally transmitted signals in the $70-250 \mathrm{MHz}$ band. This results in two measurement bands at $30-70 \mathrm{MHz}$ and 250-320 MHz.

The logarithmic power detector, used for increased receiver sensitivity, removes the high-frequency components of the received signal and produces an output which is proportional to the power in $\mathrm{dBm}$ of the signal envelope. The envelope detected signal is then ACcoupled to remove the DC offset of the detector, and scaled back to the $0-3 \mathrm{~V}$ signal range by a high-speed noninverting amplifier. Figure 3 shows a received PD signal generated by a HVPD picocoulomb PD calibrator, and the output of the logarithmic detector for the received PD pulse.

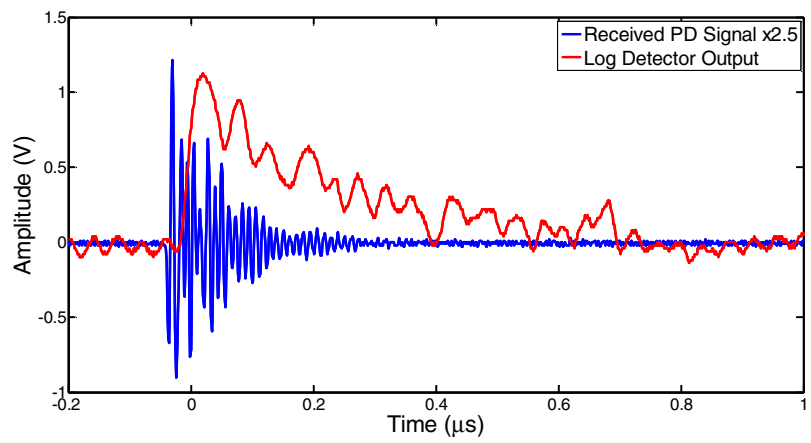

Figure 3: Received PD signal multiplied by 2.5 and logarithmic detector output.

The amplified signal is then applied to the signal conditioning section, which contains the high-speed folding ADC, a high-speed comparator and an FPGA. The comparator output is driven to a logic high level when the received $\mathrm{PD}$ event is above a set threshold level. This is applied to the FPGA, which immediately begins to clock the ADC with a bi-phase non-overlapping signal. The received $\mathrm{PD}$ signal is then sampled via the $\mathrm{ADC}$ at a rate of $25 \mathrm{MSa} / \mathrm{s}$. An internal mono-stable circuit in the FPGA ensures that sampling is performed over a $1 \mu$ s period to ensure the entire pulse is acquired. Any samples taken after the pulse cause a minimal error to the PD measurement since the output of the detector is AC-coupled. The FPGA also 'counts' the number of PD events received each time the comparator output triggers signal acquisition.

After a PD event is received and sampled, the samples and current counter value of received events are transmitted to the micro-controller. The micro-controller then performs the calculation shown in (1) and (2) in order to convert the logarithmic power samples to linear values in $\mathrm{mW}$ and calculate the total energy of the received PD.

$$
\begin{aligned}
& P_{i}=\frac{\left(\frac{V_{D E T(i)}}{A_{V(N I)}}+V_{D E T(D C)}\right)}{\text { Slope }}+P_{D E T(I N T)} \\
& E_{i}=E_{i-1}+\Delta T \sum_{i=1}^{n} 10^{\left(\frac{P_{i}}{10}\right)}
\end{aligned}
$$

where $P_{i}, V_{D E T(i)}$ and $E_{i}$ are the received power, detector output voltage and total energy at point $i$ respectively, $E_{i-1}$ is the energy at the previous data point, $\Delta T$ is the time between samples, $n$ is the number of data points, and $A_{V(N I)}$, 
$V_{D E T(D C)}$, Slope and $P_{D E T(I N T)}$ are the non-inverting amplifier voltage gain, detector DC offset voltage, detector slope and detector intercept power (in $\mathrm{dBm}$ ). The average energy is then calculated for all received pulses up to the latest received event. After a predetermined period, the average energy and event counter value are transmitted to a central HUB via the wirelessHART mote.

\section{Results}

The proposed sensor node was tested by placing the PD calibrator source approximately $1 \mathrm{~m}$ from the sensor node and transmitting a radiometric PD-like signal via a $10 \mathrm{~dB}$ attenuator pad to simulate an increased distance. The PD signal was then received and processed by the sensor. The receiving antenna and detector output were sampled at a rate of $1 \mathrm{GSa} / \mathrm{s}$ via a digital storage oscilloscope (DSO). The energies were then calculated from the sampled received PD signal, the sampled detector output and the sensor output, Figure 4. For test purposes, the sampled data was transmitted directly to a PC via a UART to USB converter. The data was then processed directly on the PC.

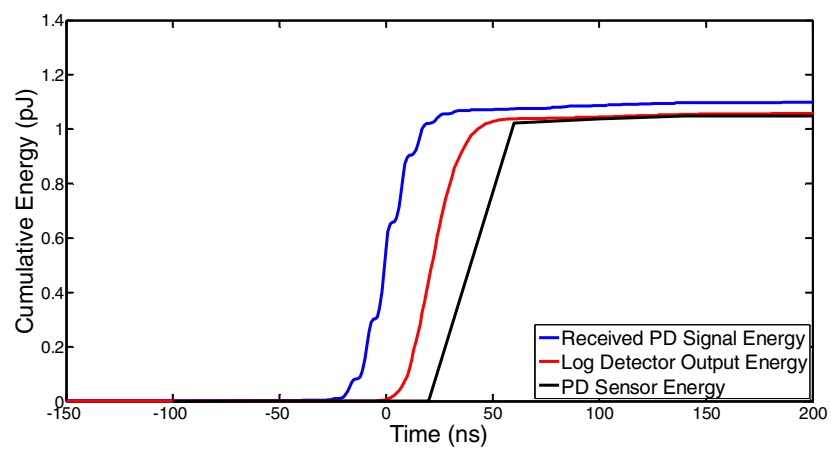

Figure 4: Calculated Energies from Sampled, Detector and Sensor Outputs.

The energies calculated via the received PD signal, the detector output and the sensor output were $1.11 \mathrm{pJ}, 1.06 \mathrm{pJ}$ and $1.04 \mathrm{pJ}$ respectively, with an error of $-0.21 \mathrm{~dB}$ between the detector output and received $\mathrm{PD}$, and an error of -0.26 $\mathrm{dB}$ between the sensor output and received $\mathrm{PD}$, which is minimal compared to the reduction in power consumption.

\section{Conclusion}

The proposed high-speed folding ADC-based PD monitoring sensor is designed to consume significant power only when a pulse is present, with a power consumption of approximately $56 \mathrm{~mW}$ from a $3.6 \mathrm{~V}$ supply when no signal is present. The sensor has been tested in a laboratory environment with an error of only $-0.26 \mathrm{~dB}$ compared to the received PD signal sampled at the antenna output.

\section{Acknowledgements}

This work was supported by the U.K. Engineering \& Physical Sciences Research Council under Grant EP/J015873.

\section{References}

1. IEC International Standard 60270, High voltage test techniques - Partial discharge measurements, $3^{\text {rd }}$ ed. Geneva: International Electrotechnical Commission (IEC), 2000, ASIN: B000XYSENS.

2. S. Birlasekaran and W. H. Leong, "Comparison of Known PD Signals with the Developed and Commercial HFCT Sensors", IEEE Transactions and Power Delivery, 22, 3, July 2007, pp. 1581-1590, doi: 10.1109/TPWRD.2007.899795.

3. G. Luo and D. Zhang, "Study on Performance of HFCT and UHF Sensors in Partial Discharge Detection" International Power \& Energy Conference (IPEC), pp. 630-635, Singapore, October 2010, doi: 10.1109/IPECON.2010.5697070.

4. F. Álvarez, F. Garnacho, J. Ortego and M. A. SánchezUrán, "Application of HFCT and UHF Sensors in On-Line Partial Discharge Measurements for Insulation Diagnosis of High Voltage Equipment", Sensors, 15, 4, March 2015, pp. 7360-7387, doi: 10.3390/s150407360.

5. N. Davies, Y. Tian, J. C. Y. Tang and P. Shiel, "NonIntrusive Partial Discharge Measurements of MV Switchgears", International Conference on Condition Monitoring and Diagnosis, pp. 1-4, Beijing, China, April 2008, doi: 10.1109/CMD.2008.4580307.

6. A. J. Reid, M. D. Judd and G. Duncan, "Simultaneous Measurement of Partial Discharge using TEV, IEC60270 and UHF Techniques", IEEE International Symposium on Electrical Insulation (ISEI), pp. 439-442, San Juan, USA, June 2012, doi: 10.1109/ELINSL.2012.6251506.

7. A. A. Jaber, P. I. Lazaridis, M. Moradzadeh, I. A. Glover, Z. D. Zaharis, M. F. Q. Vieira, M. D. Judd and R. C. Atkinson, "Calibration of Free-Space Radiometric Partial Discharge Measurements", IEEE Transactions on Dielectrics and Electrical Insulation, 24, 5, October 2017, pp. 3004-3014, doi: 10.1109/TDEI.2017.006730.

8. P. J. Moore, I. Portugues and I. A. Glover, "A nonintrusive partial discharge measurement system based on RF technology," IEEE Power Engineering Society General Meeting, pp. 628-633, Toronto, Canada, doi: 10.1109/PES.2003.1270372.

9. M. D. Judd, "Radiometric Partial Discharge Detection", International Conference on Condition Monitoring and Diagnosis, pp. 1025-1030, Beijing, China, April 2008, doi: 10.1109/CMD.2008.4580457.

10. I. E. Portugues, P. J. Moore, I. A. Glover, C. Johnstone, R. H. McKosky, M. B. Goof and L. van der Zel, "RFBased Partial Discharge Early Warning System for AirInsulated Substations", IEEE Transactions on Power Delivery, 24, 1, January 2009, pp. 20-29, doi: 10.1109/TPWRD.2008.2005464. 
11. G. Robles, J. M. Fresno, M. Sánchez-Fernández and J. Martínez-Tarifa, "Antenna Deployment for the Localization of Partial Discharge in Open-Air Substations", Sensors, 16, 4, pp. 9882-9898, doi: 10.3390/s16040541.

12. M. X. Zhu, Y. B. Wang, Y. Li, H. B. Deng, X. J. Shao and G. J. Zhang, "Detection and Localization of Partial Discharge in Air-Insulated Substations using UHF Antenna Array", $3^{\text {rd }}$ IEEE Conference on Power Engineering and Renewable Energy (ICPERE), pp. 221224, Yogyakarta, Indonesia, November 2016, doi: 10.1109/ICPERE.2016.7904875.

13. M. X. Zhu, Y. B. Wang, Q. Liu, J. N. Zhang, J. B. Deng, G. J. Zhang, X. J. Shao and W. L. He, "Localization of Multiple Partial Discharge Sources in Air-Insulated Substation Using Probability-Based Algorithm", IEEE Transactions on Dielectrics and Electrical Insulation, 24, 1, pp. 157-166, February 2017, doi: 10.1109/TDEI.2016.005964.

14. P. C. Baker, B. Stephen, M. D. Judd and S. D. J. McArthur, "Development of an Integrated Low-Power RF Partial Discharge Detector", IEEE Electrical Insulation Conference (EIC), pp. 273-277, Montreal, Canada, June 2009, doi: 10.1109/EIC.2009.5166356.

15. P. C. Baker, M. D. Judd and S. D. J. McArthur, "A Frequency-based RF Partial Discharge Detector for Lowpower Wireless Sensing", IEEE Transactions on Dielectrics and Electrical Insulation, 17, 1, pp. 133-140, February 2010, doi: 10.1109/TDEI.2010.5412011.

16. J. M. R. de Souza Neto, E. C. T. de Macedo, J. S. da Rocha Neto, E. G. Da Costa, S. A. Bhatti and I. A. Glover, "Partial Discharge Location using Unsynchronized Radiometer Network for Condition Monitoring in $\mathrm{HV}$ Substations", Journal of Physics: Conference Series, 364, 1, pp. 1-11, June 2012, doi: 10.1088/17426596/364/1/012053.

17. J. M. R. de Souza Neto, E. C. T. de Macedo, J. S. da Rocha Neto, M. F. Q. Vieira, I. A. Glover, M. D. Judd, R. Atkinson and J. Soraghan, "Plausibility of Incoherent Detection for Radiometric Monitoring of Insulation Integraity in HV Substations", Loughborough Antenna \& Propagation Conference, pp. 1-6, Loughborough, UK, November 2013, doi: 978-1-4799-0091-6.

18. Y. Zhang, D. Upton, A. Jaber, H. Ahmed, B. Saeed, P. Mather, P. Lazaridis, A. Mopty, C. Tachtatzis, R. Atkinson, M. Judd, M. F. Q. Vieira and I. Glover, "Radiometric Wireless Sensor Network Monitoring of Partial Discharge Sources in Electrical Substations", International Journal of Distributed Sensor Networks, 11, 9, pp. 1-9, January 2015, doi: 10.1155/2015/438302.

19. D. W. Upton, B. I. Saeed, U. Khan, A. Jaber, H. Mohammed, K. Mistry, P. J. Mather, P. Lazaridis, M. F. Q. Vieira, R. Atkinson, C. Tachtatzis, E. Iorkyase, M. Judd and I. A. Glover, "Wireless Sensor Network for Radiometric Detection and Assessment of Partial Discharge in HV Equipment", 32 ${ }^{\text {nd }}$ URSI General Assembly and Scientific Symposium (GASS), pp. 1-4,
Montreal, Canada, August 2017, doi: 10.23919/URSIGASS.2017.8104973.

20. H. Mohammed, P. Lazaridis, D. Upton, U. Khan, K. Mistry, B. Saeed, P. Mather, M. F. Q. Vieira, K. W. Barlee, D. S. W. Atkinson and I. A. Glover, "Partial Discharge Localization Based on Received Signal Strength", 23 ${ }^{\text {rd }}$ International Conference on Automation and Computing (ICAC), pp. 1-4, Huddersfield, UK, September 2017, doi: 10.23919/IConAC.2017.8082028.

21. J. M. R. de Souza Neto, J. S. da Rocha Neto, E. C. T. Macedo, I. A. Glover and M. D. Judd, "An Envelope Detector as a Trading Cost Technique for Radiometric Partial Discharge Detection", Instrumentation and Measurement Technology Conference (I2MTC), pp. 1-6, Montevideo, Uruguay, May 2014, doi: 10.1109/I2MTC.2014.6861013.

22. W. Kester, The Data Conversion Handbook, $1^{\text {st }}$ ed. Burlington: Newnes / Analog Devices, 2005, ISBN: 07506-7841-0.

23. C. M. Moreland, "An 8b 150MSample/s Serial ADC", IEEE Solid-State Circuits Conference, pp. 272-273, San Francisco, USA, February 1995, doi: 10.1109/ISSCC.1995.535552.

24. C. M. Moreland, F. Murden, M. Elliot, J. Young, M. Hensley and R. Stop, "A 14-bit 100-Msample/s Subranging ADC", IEEE Journal of Solid-State Circuits, 35, 12, pp. 1791-1798, December 2000, doi: $10.1109 / 4.890292$.

25. H. Dinc and F. Maloberti, "An 8-Bit Ripple Folding A/D Converter", International Symposium on Circuits and Systems (ISCAS), pp. 981-984, Bangkok, Thailand, May 2003, doi: 10.1109/ISCAS.2003.1205730. 